\title{
ARTICLE \\ Preclinical PK/PD model for the combinatorial use of dexamethasone and sulpiride in the treatment of breast cancer
}

\begin{abstract}
Qing-yu Yao ${ }^{1}$, Jian Li ${ }^{1}{ }^{12}$, Rong Chen ${ }^{1}$, Ye Yao', Jun-sheng Xue ${ }^{1}$, Wen-jun Chen ${ }^{1}$, Wei Lu' ${ }^{1}$ and Tian-yan Zhou ${ }^{1}$
Previous studies show that dopamine $\mathrm{D}_{2}$-like receptor (D2DR) antagonist sulpiride (SUL) enhances the antitumor efficacy of dexamethasone (DEX) in drug-resistant breast cancer involving cancer stem-like cells (CSCs). In this study, we investigated the pharmacokinetic (PK) properties of SUL in nude mice and developed a semi-mechanism PK/PD model to quantitatively characterize the synergistic effect of DEX and SUL in preclinical breast cancer xenografts. After nude mice received oral administration of a single dose of SUL $(50 \mathrm{mg} / \mathrm{kg}$, ig), plasma concentrations were assessed using LC-MS $/ \mathrm{MS}$. A two-compartment model with double firstorder absorption rate was developed to describe the PK profiles of SUL. The pharmacodynamic (PD) study was conducted in nude mice bearing human breast cancer MCF-7/Adr xenografts, which received oral administration of DEX $\left(1,8 \mathrm{mg} \cdot \mathrm{kg}^{-1} \cdot \mathrm{d}^{-1}\right)$ or SUL $(25$, $50 \mathrm{mg} \cdot \mathrm{kg}^{-1} \cdot \mathrm{d}^{-1}$ ) alone or in various combination. Tumor volumes were measured every other day. The PK model of SUL as well as that of DEX with a time-dependent clearance were integrated into the final PK/PD model both using Hill's function, where DEX exerted its antitumor efficacy by inhibiting the proliferation of tumor cells, and SUL enhanced DEX responses by decreasing the sensitivity parameter $\mathrm{EC}_{50}$. The PK/PD model was evaluated and subjected external validation. Finally, simulations were performed to predict the antitumor efficacy of DEX combined with SUL under various dose regimens, where changing dosing frequency of SUL had little effect, while the antitumor efficacy was predicted to be improved when DEX was given more frequently. The established PK/PD model in this study quantitatively characterizes the antitumor efficacy of the DEX combined with SUL as well as their synergism, and the simulations could provide reference for dose optimization of the combination in future studies.
\end{abstract}

Keywords: breast cancer; dexamethasone; sulpiride; pharmacokinetic/pharmacodynamic model; synergistic effect

Acta Pharmacologica Sinica (2019) 40:1596-1602; https://doi.org/10.1038/s41401-019-0251-7

\section{INTRODUCTION}

Breast cancer has the highest incidence among all cancers and is the second most common cause of mortality in women around the world [1]. Endocrine therapy, cytotoxic chemotherapy, and targeted therapy are widely used as medical therapeutic strategies for breast cancer patients $[2,3]$. Despite the increasing 5-year survival rate in recent years, drug resistance is still a major cause of death due to breast cancer and an important issue that remains to be addressed in cancer chemotherapy $[4,5]$.

Dexamethasone (DEX) is a commonly used synthetic glucocorticoid in clinical practice. Several studies have reported the antitumor potency of DEX in breast cancer, lung cancer, pancreatic cancer [6-9], etc. In our previous study, DEX was found to be efficacious in breast cancer either individually or in combination with other chemotherapy drugs, such as gemcitabine $[7,10]$. DEX downregulate the estrogen level by the glucocorticoid receptor-mediated induction of estrogen sulfotransferase, which has been demonstrated in both breast cancer and non-small cell lung cancer (NSCLC) [6, 8], and further results in the inhibition of tumor growth. Moreover, DEX works on preventing the activation of nuclear factor-kappa $B$ (NF-KB) [11] and inhibiting VEGF by downregulating the expression of HIF-1a [12], all of which contribute to its antitumor efficacy.

Cancer stem-like cells (CSCs) are thought to be involved in tumor drug resistance, recurrence, metastasis, and poor prognosis [13, 14]. Dopamine receptors (DRs) were found to be a potential target in CSCs, where the activation of dopamine $D_{1}$-like receptor (D1DR) [15] and antagonism of dopamine $D_{2}$-like receptor (D2DR) $[16,17]$ were both reported to be effective at suppressing CSCs and cancer therapy. Sulpiride (SUL) works as a specific D2DR antagonist in the treatment of schizophrenia [18] and is therefore considered helpful in inhibiting CSCs. We applied the combination of DEX and SUL to drug-resistant breast cancer xenografts, and the results showed that SUL prominently promoted the effect of DEX on inhibiting the growth of drug-resistant breast cancer by antagonizing D2DR, indicating the involvement of a CSC-related mechanism [19].

As shown in a previous study [10], the self-induction of CYP3A by DEX resulted in time-dependent pharmacokinetic (PK) changes in DEX over multiple doses. In addition, the double-peak absorption PKs of SUL after oral administration was found in humans; however, there have been no studies on the PKs of SUL in nude mice. Additionally, a synergistic pharmacodynamic (PD) interaction between DEX and SUL has been observed [19], which

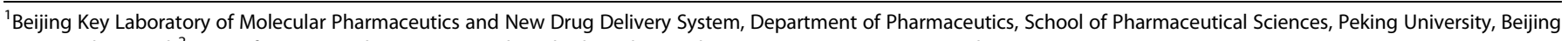
100191, China and ${ }^{2}$ Center for Drug Evaluation, National Medical Products Administration, Beijing 100022, China

Correspondence: Tian-yan Zhou (tianyanzhou@bjmu.edu.cn)

These authors contributed equally: Qing-yu Yao, Jian Li

Received: 20 March 2019 Accepted: 12 May 2019

Published online: 5 June 2019 
requires quantitative analysis and further investigation. Based on the experimental results, the aim of this study is to develop a pharmacokinetic/pharmacodynamic (PK/PD) model for the combinatorial use of DEX and SUL to quantitatively describe their antitumor efficacy and provide some insight into their synergistic interaction. Moreover, the simulation model may be helpful to optimize the dose regimen to achieve better efficacy.

\section{MATERIALS AND METHODS}

Chemicals and reagents

DEX and SUL were purchased from Sigma-Aldrich (MO, USA). RPMI-1640 medium was purchased from Macgene Biotech Co., Ltd. (Beijing, China), and fetal bovine serum (FBS) was supplied by Gibco (New York, USA). Other chemicals were obtained from Beijing Chemical Works (Beijing, China).

\section{Cell culture}

The human breast cancer cell line MCF-7/Adr was supplied by the Institute of Materia Medica, Chinese Academy of Medical Sciences (Beijing, China) as a drug-resistant breast cancer cell line. The cells were cultured in RPMI- 1640 medium with $10 \%$ FBS at $37^{\circ} \mathrm{C}$ in a $5 \%$ $\mathrm{CO}_{2}$-containing atmosphere.

\section{Animals}

Female nude mice ( $20 \pm 2 \mathrm{~g}, 4-5$ weeks of age) were purchased from Vital River (Beijing, China). The mice were housed in individual ventilated cages under standard pathogen-free conditions $\left(50 \%-60 \%\right.$ humidity, $22-24^{\circ} \mathrm{C}$, and $12 \mathrm{~h} / 12 \mathrm{~h}$ light/dark cycle). The animals were given free access to food and water, except the mice used for PK study, which were fasted for $12 \mathrm{~h}$ before administration. All animal studies were approved by the Institutional Animal Care and Use Committee of Peking University Health Science Center.

PD study

The PD study was conducted according to our previous study [19]. Briefly, MCF-7/Adr cells were subcutaneously injected into nude mice to establish an orthotopic breast cancer xenograft model. Tumor-bearing mice were divided into 11 groups $(n=5)$ and subjected to treatment 5 days after implantation. Mice in the control group were administered vehicle solutions daily. In the five monotherapy groups, mice were given SUL ( 25 or $100 \mathrm{mg} / \mathrm{kg}$, p.o.) or $\operatorname{DEX}(1,2$, or $8 \mathrm{mg} / \mathrm{kg}$, p.o.) every day. For the combination groups, mice simultaneously received DEX $(1 \mathrm{mg} / \mathrm{kg})$ with SUL $(50 \mathrm{mg} / \mathrm{kg})$ or DEX $(8 \mathrm{mg} / \mathrm{kg})$ with SUL $(25,50 \mathrm{mg} / \mathrm{kg})$. Tumor volumes were recorded every other day and calculated as follows: tumor volume $\left(\mathrm{mm}^{3}\right)=$ length $\times$ width $^{2} \times 0.5$.

PK model and simulation

The time-dependent PK model for DEX has been described in another study [10]. The PK properties of DEX were characterized by a two-compartment model with first-order absorption rate and timedependent clearance, reflecting the self-induction of CYP3A by DEX when persistently administered [20]. The PK parameter estimates and the details of the model are given in our publication [10].

An LC-MS/MS method for determining the plasma concentration of SUL in nude mice was developed and further validated. Briefly, chromatographic separation was performed on a UHPLC system (Dionex UltiMate 3000) and an API4000 QTRAP mass spectrometer (Applied Biosystems Inc., USA) was equipped with an electrospray ionization (ESI) source system. A reverse-phase Phenomenex Luna C18(2) column $(250 \times 4.60 \mathrm{~mm}, 5 \mu \mathrm{m})$ was employed. Analytes were eluted with a gradient mobile phase composed of acetonitrile and water containing $5 \mathrm{mM}$ ammonium formate (Supplementary Table S1). A $5 \mu \mathrm{L}$ working solution was added to $95 \mu \mathrm{L}$ blank plasma for calibration of standards, and quality control (QC) samples were treated with $2 \mathrm{~mL}$ of ethyl acetate and centrifuged. Then, $1.8 \mathrm{~mL}$ of the organic layer was collected and dried under nitrogen. Then, the residue was reconstituted in $120 \mu \mathrm{L}$ of methanol, and a $5 \mu \mathrm{L}$ aliquot was injected for analysis. Erlotinib was selected as an internal standard. Method validation was performed according to the US Food and Drug Administration (FDA, USA) guidelines for bioanalytical method validation (US Food and Drug Administration, 2018), including the intra- and inter-day precision and accuracy, recovery, matrix effect, and stability.

In the PK study, SUL was dissolved in 45\% ( $w / v)$ hydroxypropyl$\beta$-cyclodextrin aqueous solution and given orally at $50 \mathrm{mg} / \mathrm{kg}$ as a single dose. Blood samples were collected by extracting eyeballs at $0.5,1,1.5,2,3,5,8$, and $12 \mathrm{~h}$ after administration, and three nude mice were sacrificed at each time point. The blood samples were collected into heparinized tubes and centrifuged at $1180 \times g$ for $10 \mathrm{~min}$, and the supernatant was kept at $-20^{\circ} \mathrm{C}$. Approximately $100 \mu \mathrm{L}$ plasma samples were identically prepared from the calibration standards and QC samples.

The PK profiles of SUL were described by a two-compartment model with a double first-order absorption rate at two sites, accounting for the efflux caused by P-glycoprotein (P-gp) and reabsorption in the intestine [21]. The differential equations are as follows:

$$
\begin{aligned}
& \frac{d X_{\mathrm{a}, \text { sul }}}{d t}=-K_{\mathrm{a} 1, \text { sul }} \cdot X_{\mathrm{a}, \text { sul }} \cdot f-K_{\mathrm{a} 2, \mathrm{sul}} \cdot X_{\mathrm{a}, \mathrm{sul}} \cdot \gamma \cdot(1-f),\left\{\begin{array}{l}
\gamma=0, t<T_{\text {lag }} \\
\gamma=1, t \geq T_{\text {lag }}
\end{array}\right. \\
& X_{\mathrm{a}, \mathrm{sul}}(0)=X_{0, \text { sul }} \\
& \frac{d X_{c, \text { sul }}}{d t}=F \cdot K_{\mathrm{a} 1, \text { sul }} \cdot X_{\mathrm{a}, \mathrm{sul}} \cdot f+F \cdot K_{\mathrm{a} 2, \mathrm{sul}} \cdot X_{\mathrm{a}, \mathrm{sul}} \cdot \gamma \cdot(1-f)-X_{c, \text { sul }} \cdot \frac{C L_{\text {sul }}}{V_{\mathrm{c}, \mathrm{sul}}} \\
& -X_{c, \text { sul }} \cdot \frac{Q_{\text {sul }}}{V_{c, \text { sul }}}+X_{\mathrm{p}, \text { sul }} \cdot \frac{Q_{\text {sul }}}{V_{p, \text { sul }}}, X_{c, \text { sul }}(0)=0
\end{aligned}
$$

$\frac{d X_{\mathrm{p}, \text { sul }}}{d t}=X_{\mathrm{c}, \mathrm{sul}} \cdot \frac{Q_{\mathrm{sul}}}{V_{\mathrm{c}, \mathrm{sul}}}-X_{\mathrm{p}, \mathrm{sul}} \cdot \frac{Q_{\mathrm{sul}}}{V_{\mathrm{c}, \mathrm{sul}}}, X_{\mathrm{p}, \mathrm{sul}}(0)=0$

$C_{c, \text { sul }}=\frac{X_{c, \text { sul }}}{V_{c, \text { sul }}}$

$$
C_{p, \text { sul }}=\frac{X_{p, \text { sul }}}{V_{p, \text { sul }}}
$$

where $X_{a, \text { sul }}$ is the amount of SUL in the absorption compartment. $K_{\mathrm{a} 1, \text { sul }}$ and $K_{\mathrm{a} 2 \text {,sul }}$ indicate the first-order absorption rate at the first and second absorption sites, respectively, and $T_{\text {lag }}$ is the lag time between the two absorption. $f$ is the fraction of SUL absorbed at the first absorption site. $X_{c, \text { sul }}$ and $X_{\mathrm{p}, \text { sul }}$ represent the amount of SUL in the central and peripheral compartments, respectively. $F$ is the bioavailability of SUL. $C L_{\text {sul }}$ stands for the systematic clearance at the central compartment, while $Q_{\text {sul }}$ is the clearance between the central and peripheral compartments. $C_{c, \text { sul }}$ and $C_{p \text {,sul }}$ represent the concentrations of SUL in the central and peripheral compartments, respectively.

Simulations for multiple administration were performed based on parameter estimates. The PK profiles of SUL after multiple doses at $25 \mathrm{mg} / \mathrm{kg}$ and $50 \mathrm{mg} / \mathrm{kg}$ were simulated.

The structures of both PK models were integrated into the final PK/PD model (Fig. 1). All parameter estimates were fixed to fit the final PK/PD model.

PK/PD model construction, evaluation, and simulation The tumor natural growth model in this study was characterized by Koch et al. [22], in which tumor growth was described by an exponential growth rate followed by a linear growth rate without 


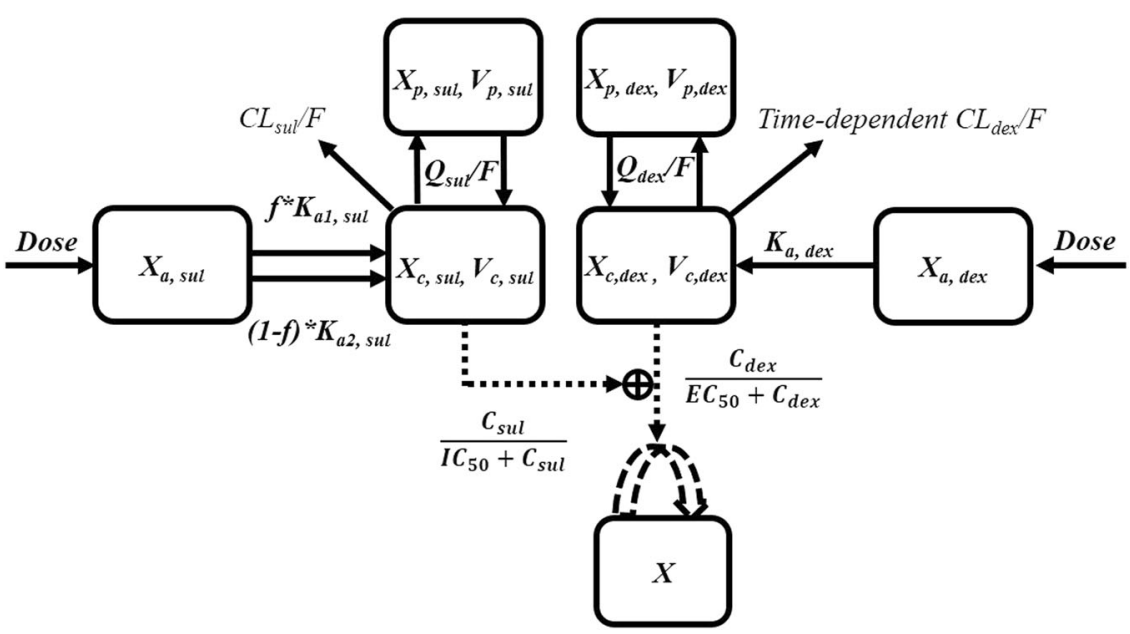

Fig. 1 A schematic representation of the PK/PD model for the combinatorial use of DEX and SUL

a threshold between the two phases. The differential equation is as follows:

$\frac{d X}{d t}=\frac{2 \cdot \lambda_{0} \cdot \lambda_{1} \cdot X}{\lambda_{1}+2 \cdot \lambda_{0} \cdot X}, X(0)=\omega_{0}$

where $X$ indicates the tumor volume, $\lambda_{0}$ and $\lambda_{1}$ stand for the exponential and linear growth rates, respectively, and $\omega_{0}$ is the initial tumor volume at the beginning of the study.

The PK/PD model was subsequently developed by adding drug effects on tumor natural growth. DEX exhibited its antitumor efficacy by inhibiting the proliferation of tumor cells [10] in the form of Hill's function instead of stimulating their eradication [7], based on its effect on decreasing estrogen and other factors involved in tumor progression, such as NF-KB and HIF-1a $[6,8,11,12]$. The differential equation is as follows [10]:

$$
\frac{d X}{d t}=\frac{2 \cdot \lambda_{0} \cdot \lambda_{1} \cdot X}{\lambda_{1}+2 \cdot \lambda_{0} \cdot X} \cdot\left(1-\frac{C_{\mathrm{dex}}}{E C_{50}+C_{\mathrm{dex}}}\right), X(0)=\omega_{0}
$$

where $C_{\text {dex }}$ is the concentration of DEX in the central compartment. $\mathrm{EC}_{50}$ represents the DEX concentration at half maximal effect, which was assumed to be $100 \%$. The parameter estimates from the model for DEX alone were then fixed to fit the combination model.

In the final PK/PD model, we assumed that SUL exerted its effect by sensitizing tumor cells to the antitumor effect of DEX with a Hill's function, as shown in Eq. 9. Another assumption is that there is no PK interaction between DEX and SUL. The structure of the combination model is shown in Fig. 1.

$$
\frac{d X}{d t}=\frac{2 \cdot \lambda_{0} \cdot \lambda_{1} \cdot X}{\lambda_{1}+2 \cdot \lambda_{0} \cdot X} \cdot\left(1-\frac{C_{\mathrm{dex}}}{E C_{50} \cdot\left(1-\frac{C_{\text {sul }}}{C_{C_{50}+C_{\text {sul }}}}\right)+C_{\mathrm{dex}}}\right), X(0)=\omega_{0}
$$

where $C_{\text {sul }}$ is the concentration of SUL in the central compartment, $I C_{50}$ represents the concentration of SUL when half of the maximal sensitizing effect (assumed as 100\%) on DEX was achieved.

Modeling and simulation in this study were conducted using NONMEM 7.2.0 (ICON Development Solutions, Ellicott City, MD, USA) and PsN 4.2.0 (Uppsala University, Uppsala, Sweden) with first-order conditional estimation with interaction (FOCEI) method. The interindividual variability (IIV) and the residual variability were assumed to follow a log-normal distribution. Exponential models were employed to characterize IIV, and a mixed error model was selected for the residual error model. Relative standard errors (RSE) were shown to evaluate the precision and reliability of the estimates. Models were evaluated and selected depending on the rationality of the estimates, change in objective function value (OFV), diagnostic plots, and visual predictive check (VPC) with 1000 simulations.

Simulations for different dosing regimens were performed based on parameter estimates of the final PK/PD model. The dosing frequencies for both DEX and SUL were changed while the total doses remained constant. In addition, the dosing schedule of DEX at $8 \mathrm{mg} / \mathrm{kg}$ given every day and SUL at $50 \mathrm{mg} / \mathrm{kg}$ administered $6 \mathrm{~h}$ later was simulated, and five nude mice were used to execute the same schedule (DEX given at 9:00 AM, SUL given at 3:00 PM, qd). The observed data were then compared with the model predictions for external validation.

Statistical analysis

The results for the PK and PD study are shown as the mean $\pm S D$, and statistical analyses were performed with GraphPad Prism 5.0 software (La Jolla, USA).

\section{RESULTS}

Pharmacokinetic model for SUL

A highly sensitive LC-MS/MS method for the quantitation of SUL in nude mouse plasma was developed and further validated. The typical multiple reaction monitoring mode chromatograms of blank nude mouse plasma, plasma samples spiked with SUL and $\mathrm{IS}$, and plasma samples collected $0.5 \mathrm{~h}$ after the administration of SUL demonstrated the specificity of the method (Supplementary Fig. S1). The calibration curves were linear within the range of $0.4-1000 \mathrm{ng} / \mathrm{mL}(y=0.00626 x+0.000867, r>0.99)$ with the lower limit of quantitation (LLOQ) of $0.4 \mathrm{ng} / \mathrm{mL}$, and a $1 / x$ weighting factor was chosen to achieve the linear regression. The precision and accuracy (Supplementary Table S2), recovery and matrix effect (Supplementary Table S3), and the stability (Supplementary Table S4) of the method were validated, and they were in accordance with the requirements of the FDA.

According to the PK profiles of SUL after a single dose at $50 \mathrm{mg} / \mathrm{kg}$, the first peak plasma concentration was observed approximately $1 \mathrm{~h}$ after administration, while the second peak was observed at $\sim 2 \mathrm{~h}$, suggesting that double absorption rates should be taken into consideration. The two-compartment model with a double first-order absorption rate fit the data well, and VPC was performed (Fig. 2a, b, Supplementary Fig. S2). The estimate of $K_{\mathrm{a} 2 \text {,sul }}$ was $538 \mathrm{~h}^{-1}$, much higher than $1.66 \mathrm{~h}^{-1}$ of $K_{\mathrm{a} 1 \text {,sul, }}$ and the $T_{\text {lag }}$ between the two absorption phases was $1.5 \mathrm{~h}$ (Table 1), which reasonably described the double peaks in plasma concentration. The $V_{c, \text { sul }} / F$ estimate $(59.6 \mathrm{~L} / \mathrm{kg})$ was much lower than $V_{p, s u} / F(392 \mathrm{~L} / \mathrm{kg})$, 

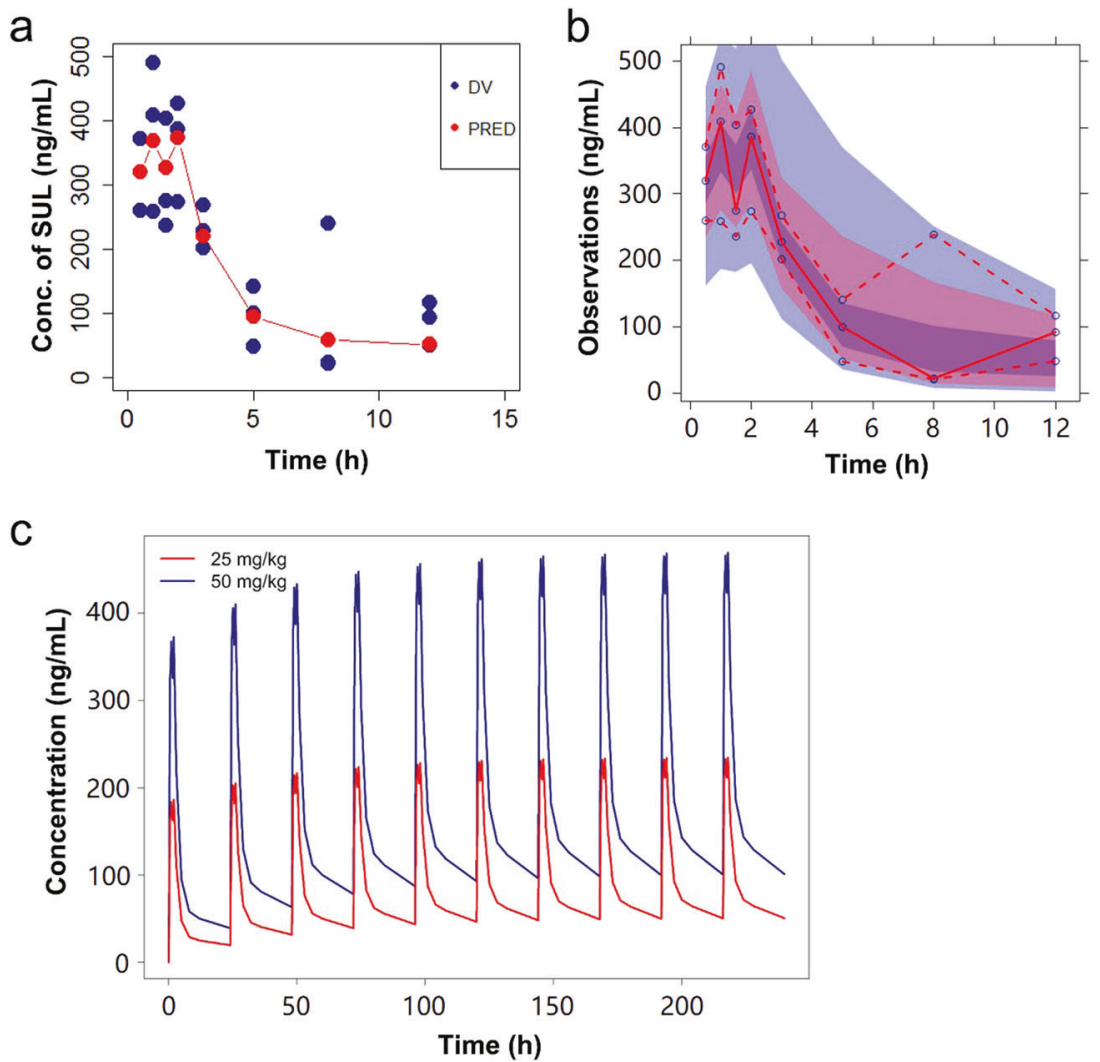

Fig. 2 a The fitness of the PK model for SUL after a single oral dose of $50 \mathrm{mg} / \mathrm{kg}$. $\mathbf{b}$ VPC results of the established PK model. The dots indicate observed data, the solid red line is the fiftieth percentile of observed data, the shaded blue area represents the $95 \% \mathrm{Cl}$ for the fifth and ninetyfifth percentiles of the predicted data, and the shaded red area represents the $95 \% \mathrm{Cl}$ for the fiftieth percentile of the predicted data. c The simulated PK profiles of SUL under different dose regimens. SUL was administered at $25 \mathrm{or} 50 \mathrm{mg} \cdot \mathrm{kg}^{-1} \cdot \mathrm{d}^{-1}$

Table 1. Parameter estimates of the PK model for SUL

\begin{tabular}{|c|c|c|c|}
\hline Parameters & Definition & Estimates (\%RSE) & IIV $(\% C V)$ \\
\hline$K_{\mathrm{a} 2, \mathrm{sul}}\left(\mathrm{h}^{-1}\right)$ & First-order absorption rate of SUL at the second site & $538(2.1)$ & 0 FIX \\
\hline$V_{c, s u l} / \mathrm{F}(\mathrm{L} / \mathrm{kg})$ & Apparent volumes of distribution in the central compartment of SUL & $59.6(24.2)$ & $0 \mathrm{FIX}$ \\
\hline$V_{\mathrm{p}, \text { sul }} / \mathrm{F}(\mathrm{L} / \mathrm{kg})$ & Apparent volumes of distribution in the peripheral compartment of SUL & $392(34.9)$ & 136.4 \\
\hline$T_{\text {lag }}(\mathrm{h})$ & Time lag between the two absorption sites & 1.50 & 0 FIX \\
\hline$\sigma_{\text {prop,sul }}(\%)$ & Proportional residual error & $19.7 \%$ & \\
\hline
\end{tabular}

which was in agreement with the results of the PK properties of SUL in humans [23]. The simulated PK profiles of SUL after multiple doses at $25 \mathrm{mg} / \mathrm{kg}$ and $50 \mathrm{mg} / \mathrm{kg}$ suggested that a steady state of SUL plasma concentration was achieved on the sixth day after the first dose when SUL was administered every day (Fig. 2c).

PK/PD model for the combination of DEX with SUL

In the final PK/PD model, the estimate of $\mathrm{IC}_{50}$ was $61.6 \mathrm{ng} / \mathrm{mL}$ within a reasonable range of SUL plasma concentrations, and the RSE of the estimates were acceptable (Table 2). In the goodnessof-fit (GOF) plots (Supplementary Fig. S3), the population predictions and individual predictions versus observation plots followed a symmetric distribution around the diagonal line, and the conditional weighted residuals were randomly distributed within the range of -4 and 4 . The individual fitting plots (Fig. 3) suggested that the individual predictions were basically in accordance with the observed data. The diagnosis plots demonstrated that the PK/PD model fit the model well.

The VPC results based on 1000 simulations are shown in Fig. 4. Most of the observations fell within the $90 \%$ confidence interval of predictions, indicating that the model had good ability to predict tumor natural growth and the antitumor effect of DEX combined with SUL.

Model simulations

Simulations for different dosing regimens were performed by fixing the PK/PD parameters. The changes in dosing frequency when the total dose of SUL was kept constant made no difference in the predicted tumor volumes (Fig. 5a), while the antitumor efficacy of the combination was predicted to be better when DEX 
Table 2. Parameter estimates of the PK/PD model for the combination of DEX and SUL

\begin{tabular}{|c|c|c|c|}
\hline Parameters & Definition & Estimates (\%RSE) & IIV (\%CV) \\
\hline$\lambda_{1}\left(\mathrm{~mm}^{3} \cdot \mathrm{h}^{-1}\right)^{\mathrm{a}}$ & Linear tumor growth rate & $28.1(28.7)$ & 21.1 \\
\hline $\mathrm{EC}_{50}(\mathrm{ng} / \mathrm{mL})^{\mathrm{a}}$ & DEX concentration when half of the maximal antitumor effect was achieved & $61.6(18.8)$ & 72.6 \\
\hline $\mathrm{IC}_{50}(\mathrm{ng} / \mathrm{mL})$ & SUL concentration when half of the maximal sensibilization effect on DEX was achieved & $61.9(36.8)$ & 138.2 \\
\hline$\sigma_{\text {prop }}(\%)$ & Proportional residual error in PK/PD model & $30 \%$ & \\
\hline
\end{tabular}

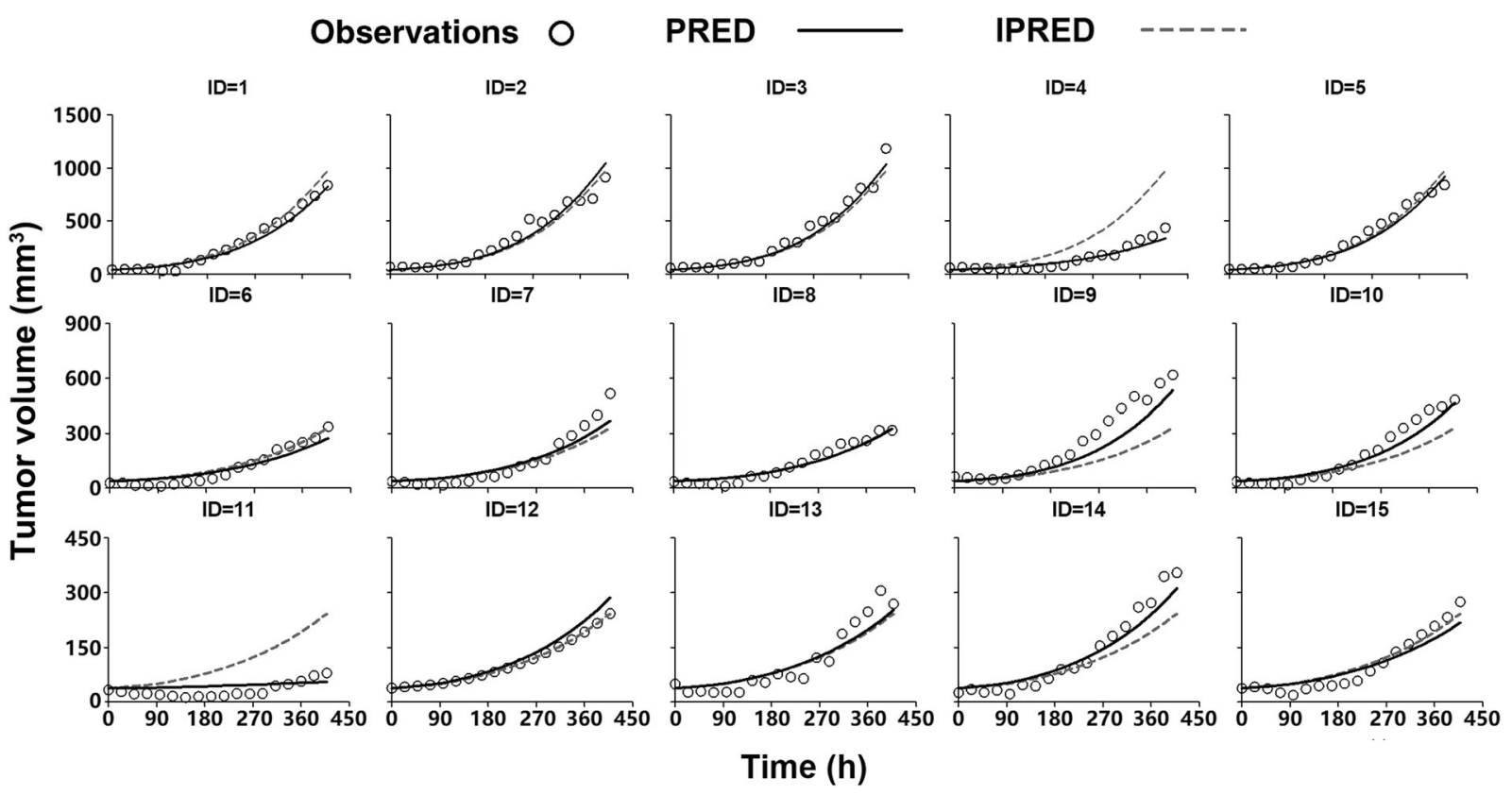

Fig. 3 Individual fits of the PK/PD model for DEX combined with SUL. ID $=1-5$, DEX $1 \mathrm{mg} \cdot \mathrm{kg}^{-1} \cdot \mathrm{d}^{-1}$ combined with SUL $50 \mathrm{mg} \cdot \mathrm{kg}^{-1} \cdot \mathrm{d}^{-1}$; ID $=$ $6-10$, DEX $8 \mathrm{mg} \cdot \mathrm{kg}^{-1} \cdot \mathrm{d}^{-1}$ combined with SUL $25 \mathrm{mg} \cdot \mathrm{kg}^{-1} \cdot \mathrm{d}^{-1} ; \mathrm{ID}=11-15$, DEX $8 \mathrm{mg} \cdot \mathrm{kg}^{-1} \cdot \mathrm{d}^{-1}$ combined with SUL $50 \mathrm{mg} \cdot \mathrm{kg}^{-1} \cdot \mathrm{d}^{-1}$. DV observed data, IPRED individual predicted tumor size, PRED predicted tumor size

was administered more frequently (Fig. 5b, c). The results suggested that increasing the dosing frequency of DEX when possible might improve its antitumor efficacy.

In addition, the dosing schedule of DEX at $8 \mathrm{mg} \cdot \mathrm{kg}^{-1} \cdot \mathrm{d}^{-1}$ combined with SUL at $50 \mathrm{mg} \cdot \mathrm{kg}^{-1} \cdot \mathrm{d}^{-1}$ administered $6 \mathrm{~h}$ after DEX was predicted and implemented in the experiment. The experimental data were in good agreement with the predictions, further confirming the predictability of the model by external validation (Fig. 5d).

\section{DISCUSSION}

The antitumor efficacy of DEX has been reported in several publications [6,9] and was further confirmed by our previous studies $[7,8]$. On the other hand, D2DR antagonists have been utilized in combination with chemotherapy drugs in lung cancer [17]. Since both DEX and SUL are commonly used clinical drugs without major safety issues, especially in cancer [18, 24], along with their low cost to patients, we investigated the antitumor efficacy of the combination in a preclinical drug-resistant breast cancer xenograft model and the underlying mechanism [19]. In this study, we mainly focused on the PK/PD model for combination therapy with DEX and SUL and its application using simulations. The PK model for SUL with the assumption of two absorption peaks was established. Thus, the effects of transporters and drug metabolizing enzymes were simultaneously considered in the combination PK/PD model. Meanwhile, the PD effect of SUL for reducing the apparent $\mathrm{EC}_{50}$ in the combination PK/PD model is completely new compared with our previous published model. Moreover, the developed PK/PD model quantitatively described the synergism between DEX and SUL and was further applied to simulations for different dosages, which could be conductive to future studies.

In the PK model for SUL, various structures were tried to capture the double peaks of plasma concentration: zero-order absorption rate alone, first-order absorption rate alone, zero-order absorption rate together with first-order absorption rate, zero-order absorption rate followed by first-order absorption rate, and double firstorder absorption rate at two sites. In addition, both onecompartment and two-compartment models were used for the structure. Finally, a two-compartment model with a double firstorder absorption rate was selected. It has been reported that SUL is the substrate of P-gp when absorbed in the gastrointestinal (GI) tract, and some SUL is pumped out by P-gp and then reabsorbed in the $\mathrm{Gl}$ [21] tract, accounting for the two peaks in the plasma concentration curve. Hence, the double absorption rate suggested that dual absorption occurred at different sites, capturing the 
process of absorption and reabsorption in the Gl tract, where $T_{\text {lag }}$ was used to describe the time lag between the dual absorption pattern, consistent with the PK model for SUL in humans [23].

In the PK/PD model for DEX combined with SUL, the effect of SUL was characterized by the enhancement of sensitivity to DEX.



Time (h)

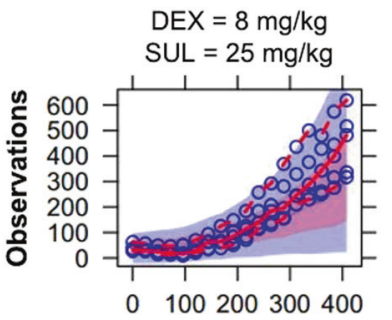

Time (h)

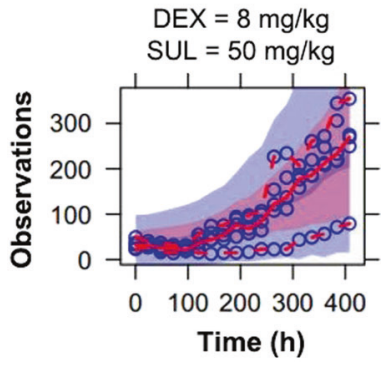

Fig. 4 The VPC results of the integrated PK/PD model for DEX combined with SUL based on 1000 simulations. The dots are the observed data, and the solid red line represents the 50th percentile of observed data; the blue shaded area represents the $95 \% \mathrm{Cl}$ for the fifth and ninty-fifth percentiles of the predicted data; and the shaded red area represents the $95 \% \mathrm{Cls}$ for the fiftieth percentile of the predicted data
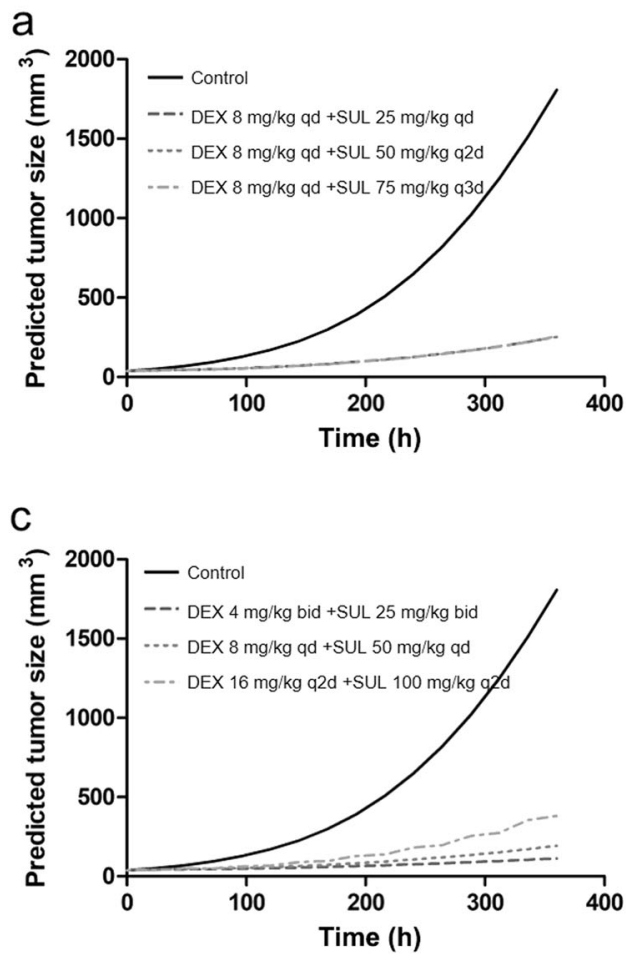

SUL showed no significant effect on tumor growth when administered alone, but the antitumor efficacy of DEX was significantly improved when combined with SUL compared to that of monotherapy with DEX at the same dose [10]. Our previous work has demonstrated that SUL significantly downregulates the CSC frequency in drug-resistant breast cancer by antagonizing D2DR, although CSCs account for only $11.27 \%$ of cells in the preclinical drug-resistant breast cancer xenograft, in addition to having a limited impact on differentiated tumor cells [19]. However, DEX exerts its antitumor effect by inhibiting the natural growth of tumor cells and is taken up by the majority of tumor cells except for CSCs $[6,10]$. Thus, the combination of DEX and SUL shows a good synergistic effect, and the parameter $\mathrm{IC}_{50}$ indicates the additive effect of SUL on the $\mathrm{EC}_{50}$, indicating the sensitivity of tumor natural growth to DEX. The effects of both DEX and SUL were characterized by Hill's function with the $E_{\max }\left(\right.$ or $I_{\max }$ ) fixed to 1 , because the model failed to perform well when $E_{\max }$ (or $I_{\max }$ ) was estimated, possibly due to limitations in the observed data. Then, assumptions were made that tumor growth would be completely inhibited and remain stagnant when the maximum effect of DEX is achieved and that its sensitivity to DEX would be maximized when the maximum effect of SUL is reached, which is also seen in other publications $[25,26]$.

Although SUL was found to be effective against CSCs by antagonizing D2DR, agonists of D1DR may play a similar role, as both classes of molecules might result in an increase in cellular cyclic adenosine monophosphate (CAMP) levels and decrease in the activity of protein kinase A (PKA) [27, 28]. In our previous study, dopamine was found to downregulate the CSC frequency by activating D1DR in breast cancer [15]; PK/PD models were developed for dopamine combined with sunitinib in lung cancer [29] and combined with axitinib in breast cancer [30]. An on/off effect model, regardless of PK, was employed to describe the effect of dopamine on the antitumor efficacy of sunitinib or axitinib, as the half-life of dopamine in animals or humans is fairly short [31]. b

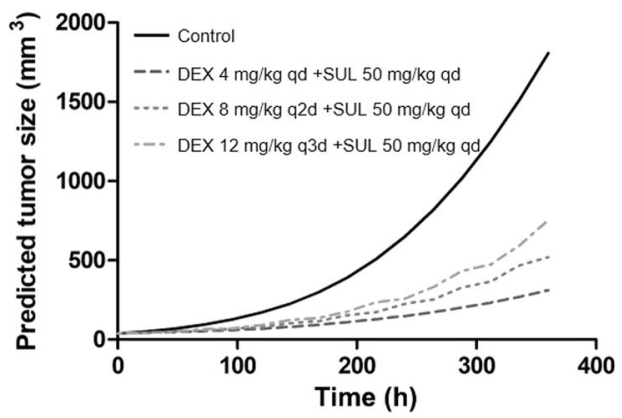

d

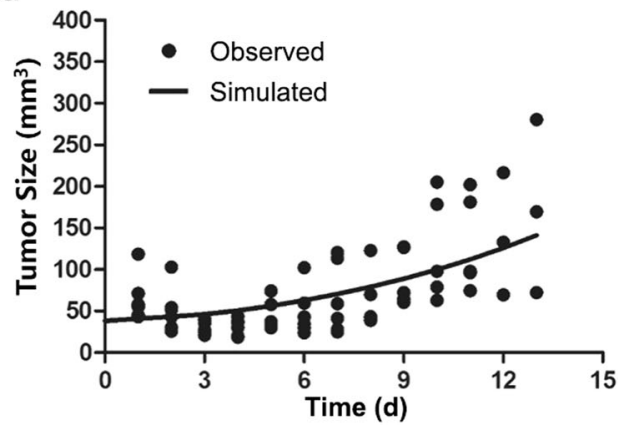

Fig. 5 Simulated tumor growth kinetics under different dose regimens. The dose schedules are presented in related legends (a, $\mathbf{b}$, and $\mathbf{c}$ ). External validation of the PK/PD model with the dosing schedule of $8 \mathrm{mg} \cdot \mathrm{kg}^{-1} \cdot \mathrm{d}^{-1}$ DEX and $50 \mathrm{mg} \cdot \mathrm{kg}^{-1} \cdot \mathrm{d}^{-1} \mathrm{SUL}$ given in $6-\mathrm{h}$ intervals. The solid line represents the simulated tumor size, and the dots represent the observed tumor sizes (d) 
Moreover, dopamine was found to continuously enhance the effects of sunitinib or axitinib for 3 days after a single dose [29, 30], while SUL in this study was given every day to maintain the efficacy. The different dosing frequencies might be attributed to the patterns of action of the two agents, because dopamine acts as an agonist activating signaling pathways, but SUL is an antagonist that mainly works by inhibiting D2DR [32]. Thus, a steady plasma concentration of SUL might be needed to maintain the function. Nevertheless, dopamine can only be administered via intravenous routes [33], but SUL can be given orally and thus can result in better compliance in clinical application.

Simulations of various dosing schedules were performed and further compared. The dosing frequency of SUL did not show a significant impact on the antitumor efficacy of the combination treatment, but changes in DEX dosing resulted in different outcomes. Basically, a lower dose administered more frequently to achieve the total dose was found to lead to better antitumor efficacy. In addition, the dosing schedule of DEX at $8 \mathrm{mg} \cdot \mathrm{kg}^{-1} \cdot \mathrm{d}^{-1}$ combined with SUL at $50 \mathrm{mg} \cdot \mathrm{kg}^{-1} \cdot \mathrm{d}^{-1}$ administered $6 \mathrm{~h}$ after DEX was simulated, and it matched with the external validation data well, which further confirmed the predictive ability of the model. However, the sequential dosing schedule of DEX and SUL seemed to have similar antitumor efficacy with the simultaneous schedule, suggesting the lack of additional benefit.

In summary, a semi-mechanism-based PK/PD model was developed and evaluated based on preclinical data to quantitatively describe the antitumor efficacy of DEX combined with SUL and their synergistic effect in drug-resistant breast cancer. DEX inhibited tumor natural growth, and SUL significantly enhanced the efficacy of DEX. A simulation study demonstrated that a better efficacy could be achieved when DEX was given more frequently, which could be conductive to future studies.

\section{ACKNOWLEDGEMENTS}

The study was supported by the National Natural Science Foundation of China (NSFC) (Grant No. 81673500). The first two authors are supported by Pfizer Sponsorships for Pharmacometrics.

\section{AUTHOR CONTRIBUTIONS}

TYZ, WL, and JL designed research; JL, QYY, YY, JSX, and WJC performed research; JL, QYY, and RC analyzed the data; QYY and TYZ organized the manuscript.

\section{ADDITIONAL INFORMATION}

The online version of this article (https://doi.org/10.1038/s41401-019-0251-7) contains supplementary material, which is available to authorized users.

Competing interests: The authors declare no competing interest.

\section{REFERENCES}

1. Siegel RL, Miller KD, Jemal A. Cancer statistics, 2019. CA Cancer J Clin. 2019;69:7-34.

2. Gradishar WJ, Anderson BO, Balassanian R, Blair SL, Burstein HJ, Cyr A, et al. Invasive Breast Cancer Version 1.2016, NCCN Clinical Practice Guidelines in Oncology. J Natl Compr Canc Netw. 2016;14:324-54. (PMID: 26957618).

3. Waks AG, Winer EP. Breast cancer treatment: a review. JAMA. 2019;321:288-300.

4. Gu G, Dustin D, Fuqua SA. Targeted therapy for breast cancer and molecular mechanisms of resistance to treatment. Curr Opin Pharm. 2016;31:97-103.

5. Bai X, Ni J, Beretov J, Graham P, Li Y. Cancer stem cell in breast cancer therapeutic resistance. Cancer Treat Rev. 2018;69:152-63.

6. Gong $\mathrm{H}$, Jarzynka MJ, Cole $\mathrm{TJ}$, Lee $\mathrm{JH}$, Wada $\mathrm{T}$, Zhang B, et al. Glucocorticoids antagonize estrogens by glucocorticoid receptor-mediated activation of estrogen sulfotransferase. Cancer Res. 2008;68:7386-93.

7. Yuan Y, Zhou X, Ren Y, Zhou S, Wang L, Ji S, et al. Semi-mechanism-based pharmacokinetic/pharmacodynamic model for the combination use of dexamethasone and gemcitabine in breast cancer. J Pharm Sci. 2015;104:4399-408.
8. Wang LJ, Li J, Hao FR, Yuan Y, Li JY, Lu W, et al. Dexamethasone suppresses the growth of human non-small cell lung cancer via inducing estrogen sulfotransferase and inactivating estrogen. Acta Pharm Sin. 2016;37:845-56.

9. Egberts JH, Schniewind B, Patzold M, Kettler B, Tepel J, Kalthoff H, et al. Dexamethasone reduces tumor recurrence and metastasis after pancreatic tumor resection in SCID mice. Cancer Biol Ther. 2008;7:1044-50.

10. Li J, Chen R, Yao QY, Liu SJ, Tian XY, Hao CY, et al. Time-dependent pharmacokinetics of dexamethasone and its efficacy in human breast cancer xenograft mice: a semi-mechanism-based pharmacokinetic/pharmacodynamic model. Acta Pharm Sin. 2018;39:472-81.

11. Castro-Caldas M, Mendes AF, Carvalho AP, Duarte CB, Lopes MC. Dexamethasone prevents interleukin-1beta-induced nuclear factor-kappaB activation by upregulating IkappaB-alpha synthesis, in lymphoblastic cells. Mediat Inflamm. 2003;12:37-46.

12. Yano A, Fujii Y, Iwai A, Kageyama Y, Kihara K. Glucocorticoids suppress tumor angiogenesis and in vivo growth of prostate cancer cells. Clin Cancer Res. 2006;12:3003-9.

13. Ablett MP, Singh JK, Clarke RB. Stem cells in breast tumours: are they ready for the clinic? Eur J Cancer. 2012;48:2104-16.

14. Zhao J. Cancer stem cells and chemoresistance: the smartest survives the raid. Pharm Ther. 2016;160:145-58.

15. Wang S, Mou Z, Ma Y, Li J, Li J, Ji X, et al. Dopamine enhances the response of sunitinib in the treatment of drug-resistant breast cancer: Involvement of eradicating cancer stem-like cells. Biochem Pharm. 2015;95:98-109.

16. Sachlos E, Risueno RM, Laronde S, Shapovalova Z, Lee JH, Russell J, et al. Identification of drugs including a dopamine receptor antagonist that selectively target cancer stem cells. Cell. 2012;149:1284-97.

17. Yeh CT, Wu AT, Chang PM, Chen KY, Yang CN, Yang SC, et al. Trifluoperazine, an antipsychotic agent, inhibits cancer stem cell growth and overcomes drug resistance of lung cancer. Am J Respir Crit Care Med. 2012;186:1180-8.

18. Rzewuska M. [Sulpiride: the best known atypical, safe neuroleptic drug. Review of literature]. Psychiatr Pol. 1998;32:655-66.

19. Li J, Yao QY, Xue JS, Wang LJ, Yuan Y, Tian XY, et al. Dopamine D2 receptor antagonist sulpiride enhances dexamethasone responses in the treatment of drug-resistant and metastatic breast cancer. Acta Pharm Sin. 2017;38:1282-96.

20. Iwanaga K, Honjo T, Miyazaki M, Kakemi M. Time-dependent changes in hepatic and intestinal induction of cytochrome P450 3A after administration of dexamethasone to rats. Xenobiotica. 2013;43:765-73.

21. Watanabe K, Sawano T, Jinriki T, Sato J. Studies on intestinal absorption of sulpiride (3): intestinal absorption of sulpiride in rats. Biol Pharm Bull. 2004;27:77-81.

22. Koch G, Walz A, Lahu G, Schropp J. Modeling of tumor growth and anticancer effects of combination therapy. J Pharm Pharm. 2009;36:179-97.

23. Helmy SA. Therapeutic drug monitoring and pharmacokinetic compartmental analysis of sulpiride double-peak absorption profile after oral administration to human volunteers. Biopharm Drug Dispos. 2013;34:288-301.

24. Leggas $M$, Kuo KL, Robert F, Cloud G, deShazo M, Zhang $R$, et al. Intensive antiinflammatory therapy with dexamethasone in patients with non-small cell lung cancer: effect on chemotherapy toxicity and efficacy. Cancer Chemother Pharm. 2009;63:731-43.

25. Lim CN, Salem AH. A semi-mechanistic integrated pharmacokinetic/pharmacodynamic model of the testosterone effects of the gonadotropin-releasing hormone agonist leuprolide in prostate cancer patients. Clin Pharm. 2015;54: 963-73.

26. Romero E, Velez de Mendizabal N, Cendros JM, Peraire C, Bascompta E, Obach R, et al. Pharmacokinetic/pharmacodynamic model of the testosterone effects of triptorelin administered in sustained release formulations in patients with prostate cancer. J Pharm Exp Ther. 2012;342:788-98.

27. Beaulieu JM, Gainetdinov RR. The physiology, signaling, and pharmacology of dopamine receptors. Pharm Rev. 2011;63:182-217.

28. Missale C, Nash SR, Robinson SW, Jaber M, Caron MG. Dopamine receptors: from structure to function. Physiol Rev. 1998;78:189-225.

29. Hao F, Wang S, Zhu X, Xue J, Li J, Wang L, et al. Pharmacokineticpharmacodynamic modeling of the anti-tumor effect of sunitinib combined with dopamine in the human non-small cell lung cancer xenograft. Pharm Res. 2017;34:408-18.

30. Ma YH, Wang SY, Ren YP, Li J, Guo TJ, Lu W, et al. Antitumor effect of axitinib combined with dopamine and PK-PD modeling in the treatment of human breast cancer xenograft. Acta Pharm Sin. 2019;40:243-56.

31. Bhatt-Mehta V, Nahata MC. Dopamine and dobutamine in pediatric therapy. Pharmacotherapy. 1989;9:303-14.

32. O'Connor SE, Brown RA. The pharmacology of sulpiride-a dopamine receptor antagonist. Gen Pharm. 1982;13:185-93.

33. Dasta JF, Kirby MG. Pharmacology and therapeutic use of low-dose dopamine. Pharmacotherapy. 1986;6:304-10. 